\title{
Fronts between Hopf- and Turing-type domains in a two-component reaction-diffusion system
}

\author{
G. Heidemann, M. Bode and H.-G. Purwins \\ Institut für Angewandte Physik, Universität Münster, Corrensstrasse 2/4, W-4400 Münster, Germany
}

Received 12 November 1992; revised manuscript received 30 March 1993; accepted for publication 19 April 1993 Communicated by A.R. Bishop

\begin{abstract}
Propagating and standing fronts between Hopf- and Turing-type domains are observed experimentally on a one-dimensional array of resistively coupled nonlinear LC-oscillators describable by a two-component reaction-diffusion equation. Numerical and experimental results are compared in particular with respect to front velocities. In the neighbourhood of a codimension-two point two coupled Ginzburg-Landau equations, derived by multiple scale methods, are a useful approximation.
\end{abstract}

Fronts have always attracted much interest since they can mediate between two relatively simple states of a system and by this way give access to more complicated structures. We investigate fronts on an electrical network consisting of 128 identical LC-oscillators with an S-shaped nonlinearity $S(I)$, similar to Bonhoeffer-van der Pol oscillators [1]. The oscillators ("cells") are coupled via resistors (fig. 1). The network equations in the discrete variables $I_{i}, U_{i}$ for current and voltage of the $i$ th cell can be approximated by two differential equations in the continuous dimensionless variables $v(x, t), w(x, t)$,

$$
i=D_{v} \Delta v+f(v)-w, \quad f(v)=\lambda v-v^{3} \text {, }
$$$$
\delta \dot{w}=D_{w} \Delta w+v-w-\kappa_{1} \text {. }
$$

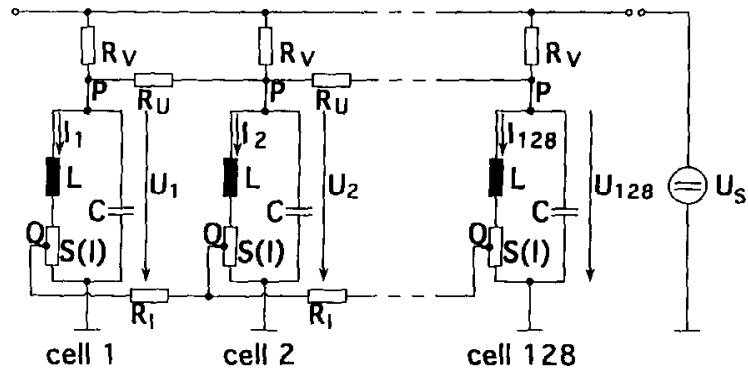

Fig. 1. Circuit diagram of the electrical network, consisting of $N=128$ oscillators. Parameters: $L=33 \mathrm{mH}, C=10-50 \mathrm{nF}, R_{l}=47$ $\mathrm{k} \Omega, R_{U}=140-650 \Omega, R_{V}=3 \mathrm{k} \Omega, U_{S}=17.56 \mathrm{~V}$.
The parameters are real with $D_{v}, D_{w}, \delta, \lambda>0 . v\left(x_{i}\right)$ and $w\left(x_{i}\right)$ are simple linear transformations of $I_{i}$ and $U_{i}$, respectively,

$v\left(x_{i}\right)=\alpha\left(I_{i}-I^{*}\right), \quad w\left(x_{i}\right)=\beta\left(U^{*}-U_{i}\right)$,

$\alpha, \beta, I^{*}, U^{*}=$ const

System (1) is a reaction-diffusion system of activator-inhibitor type as studied by Turing [2]. The derivation of this continuous system written in normalized variables and parameters from the network equations is outlined in ref. [1], here we give only the basic transformations. The S-shaped voltagecurrent characteristic $S(I)$ is approximated by

$S(I) \approx U^{*}-\chi\left(I-I^{*}\right)+\varphi\left(I-I^{*}\right)^{3}$,

$\chi=1270 \Omega, \varphi=0.165 \mathrm{~V} /(\mathrm{mA})^{3}$,

$I^{*}=3.34 \mathrm{~mA}, \quad U^{*}=7.54 \mathrm{~V}$.

The normalized version of $S(I)$, its cubic approximation and the load line are given in fig. 2. The transformations between the experimental and dimensionless parameters are

$$
\begin{aligned}
& D_{v}=\frac{\gamma}{R_{V} R_{I}} \frac{\xi^{2}}{N^{2}}, \quad D_{w}=\frac{R_{V}}{R_{U}} \frac{\xi^{2}}{N^{2}}, \\
& \delta=\frac{R_{V}^{2} C}{L}, \quad \lambda=\frac{\chi}{R_{V}}, \quad \kappa_{1}=\alpha\left(\frac{U_{S}-U^{*}}{R_{V}}-I^{*}\right),
\end{aligned}
$$




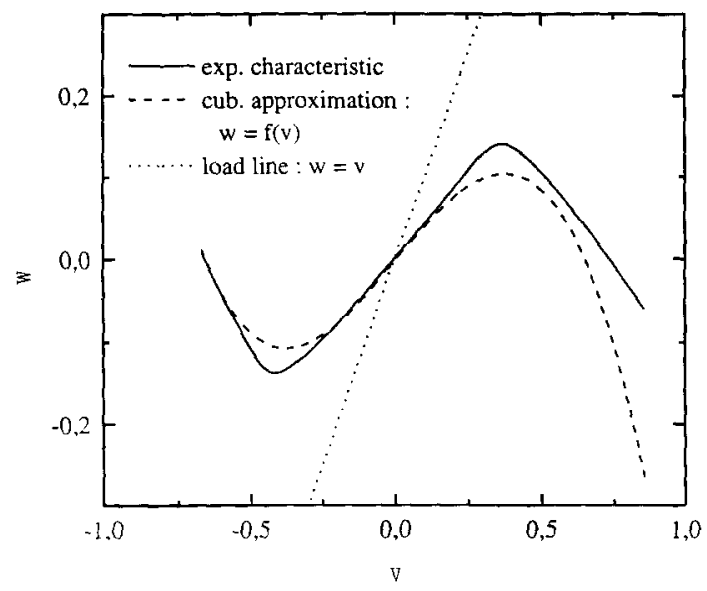

Fig. 2. Voltage-current characteristic of the nonlinear resistance $S(I)$ in the normalized variables $v$ and $w$, its cubic approximation $f(v)$ and the load line for $\kappa_{1}=0$ intersecting $S(I)$ at the inflection point.

where $\xi=$ length of the system, $N=$ number of cells, $\alpha=\sqrt{\varphi / R_{V}}, \quad \beta=\sqrt{\varphi / R_{V}^{3}}, \quad \gamma=11.5 \times 10^{6} \Omega^{2}$.

Also the time is rescaled: $t_{\text {dimensionless }}=\left(R_{V} / L\right)$ $X t_{\text {experimental }}$.

In semiconductors and gas discharge systems many pattern formation phenomena can be described qualitatively by equations of this type $[3,4]$.

Hence we fix the values of $\lambda=0.423, D_{w}=$ $2.88 \times 10^{-3}$ and $\kappa_{1}=0$ but vary $D_{v}$ and $\delta$, so the single cell has only one stationary state at $v=w=0$. If $\lambda \delta>1$, this stationary state is unstable, and a stable limit cycle exists at least in the space clamped system. With zero flux Neumann-type boundary conditions and if the voltage $U_{S}$ is switched on rapidly enough, the whole network is found to oscillate almost homogeneously with $3.5-6.8 \mathrm{kHz}$ for $\delta=13.3$ 3.6. Now, applying a constant external voltage to one boundary, a front can be started, propagating through the system and leaving behind a spatially periodic stationary state similar to Turing-type structures [1,5] (figs. 3-5). This final stable structure undergoes only minor modifications near the boundary when the constant boundary voltage is switched off and the Neumann-type boundary condition is reestablished.

Such fronts will be called "freezing fronts" (FF). The front velocity $c$ decreases with increasing $D_{v}$ and
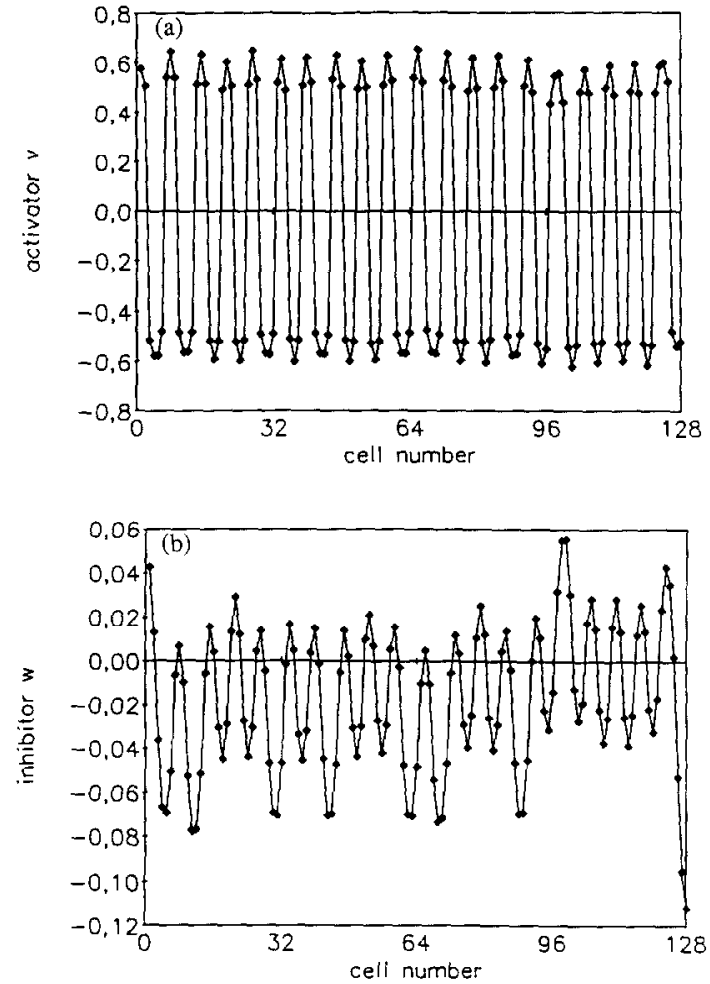

Fig. 3. Experimentally obtained Turing-type domain on the network left behind by a "freezing front" (FF). $D_{\nu}=1.12 \times 10^{-5}$, $\delta=9.95, \xi=1.5$. The wavelength of the pattern is $A=6.6$ cells The corresponding Turing wavelength is $A_{\mathrm{T}}=7.4$ cells, resulting from $A_{\mathrm{T}}=2 \pi / q_{\mathrm{T}}, q_{\mathrm{T}}^{2}=1 / \sqrt{D_{v} D_{w}}-1 / D_{w}$ and a conversion factor of $N / \xi=128 / 1.5$ cells. Taking into account the discreteness of the system leads to $A_{\mathrm{T}, \mathrm{dis}}=7.2$ cells. (a) Activator $v$, (b) inhibitor $w$.

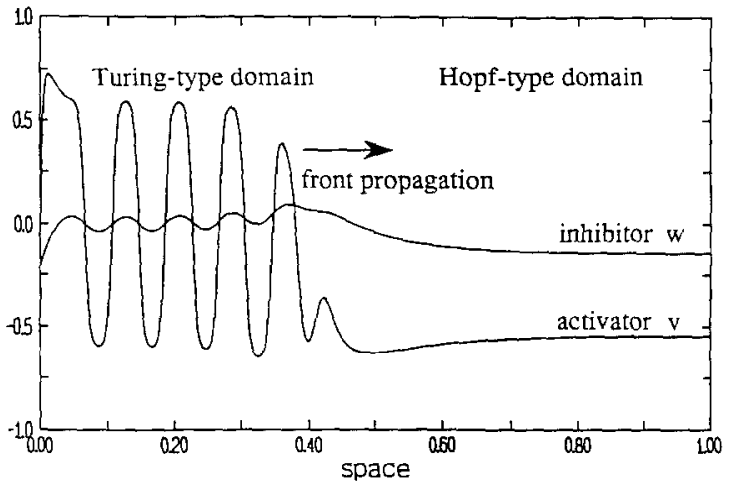

Fig. 4. Numerical computation of a FF started at the left boundary. $D_{v}=10^{-5}, \delta=9.0, A=0.079, A_{\mathrm{T}}=0.084$. 


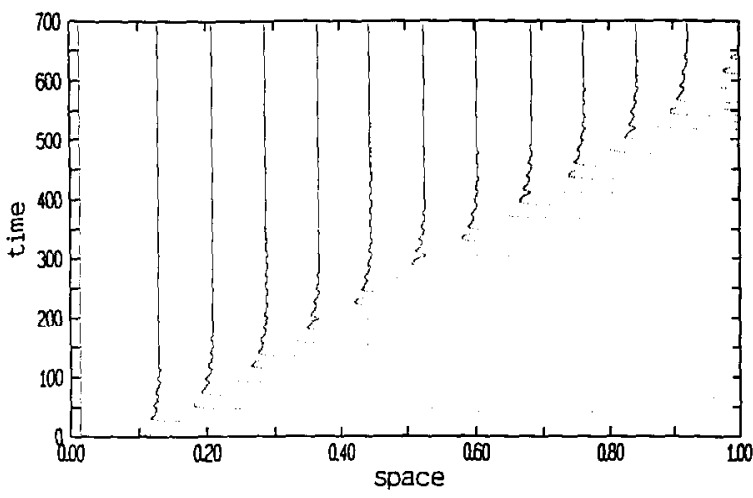

Fig. 5. The propagation of a FF in space and time can be illustrated by the generation of the peaks of the Turing-type domain. In this diagram a point is set at the peaks of the activator distribution for each time step. The parameters for this numerical computation are the same as in fig. 4 .

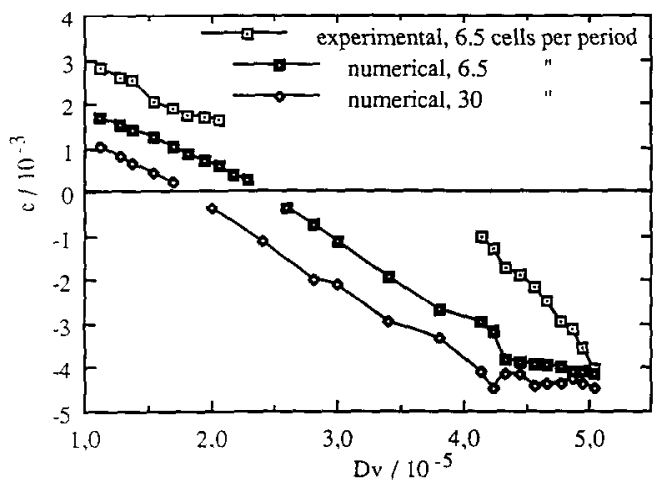

Fig. 6. Front velocities $c$ at $\delta=9.95$ plotted as a function of $D_{\boldsymbol{v}} \mathrm{A}$ positive velocity corresponds to a FF, a negative one to a $M F$.

even takes negative values (fig. 6). To observe negative velocities, first a stationary Turing-type domain must be prepared for example by a FF at lower $D_{v}$, then $D_{v}$ is increased and some additional oscillating cells are connected with one boundary. The latter induces a "melting front" (MF), which travels through the network generating a homogeneously oscillating state (Hopf-type domain). In fig. 6 there is a gap between the branches of positive and negative velocities. Within the gap FFs or MFs travel only through a part of the system until they are pinned at some position.

Front velocities computed numerically with a spatial discretization of 128 points, that is 6.5 points per period, match reasonably well with experimentally observed values, considering that the nonlinearity $f(v)$ used in the calculations is only an approximation to the real characteristic $S(I)$. Improving the numerical discretization to about 30 points per spatial period and 500 in total, smaller velocities are obtained. What is more, FFs at different $D_{\nu}$ now produce Turing-type domains which differ in wavelength, whereas with lesser discretization all FFs result in the same structure. So there are now several MF branches, one branch to each structure left behind by a FF (fig. 7). Higher spatial discretizations do not change the results any more, so 30 points per period are used for all further numerical investigations. The velocities of FFs decrease with increasing $\delta$. The dependence of $c$ on both parameters can be shown by means of its zeros in the $D_{v}-1 / \delta$ plane (fig. 8).

FFs and MFs can be understood as transitions between two competing domains. An analytical approach can be made if we choose $D_{v}$ and $\delta$ close to the codimension-two bifurcation point $D_{v, \mathrm{c}}=$ $1.67 \times 10^{-4}, \delta_{\mathrm{c}}=2.36$, where the leading Hopf and Turing bifurcation of (1) coincide. Both bifurcations are of supercritical type leading to stable structures, so if for example $\delta$ is fixed at $\delta_{\mathrm{c}}$, a Turing-type structure of wavenumber $k_{\mathrm{c}}$ emanates from the homogeneous stationary state $v(x)=w(x)=0$ when $D_{\nu}$

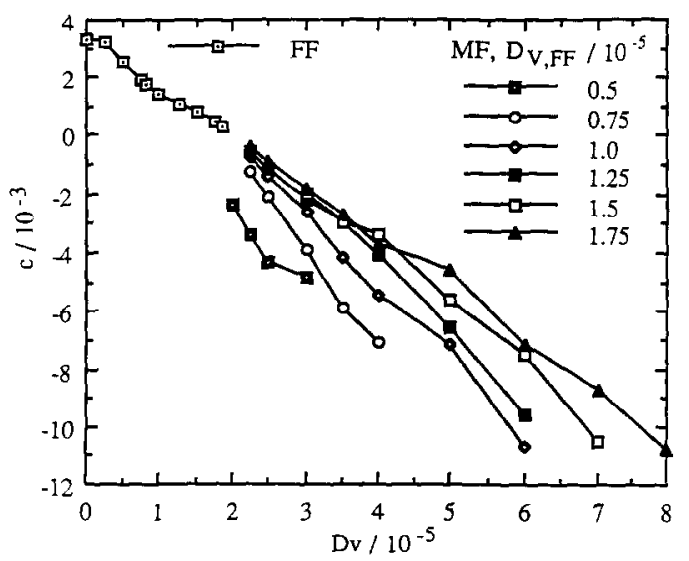

Fig. 7. Numerically computed front velocities at $\delta=9.0$. There is one branch of FFs, but several branches of MFs, indicated by different plotting symbols. Each MF branch belongs to fronts between the homogeneous Itopf-type domain and a Turing-type domain which was left behind by a FF at $D_{\nu, \mathrm{FF}}$. 


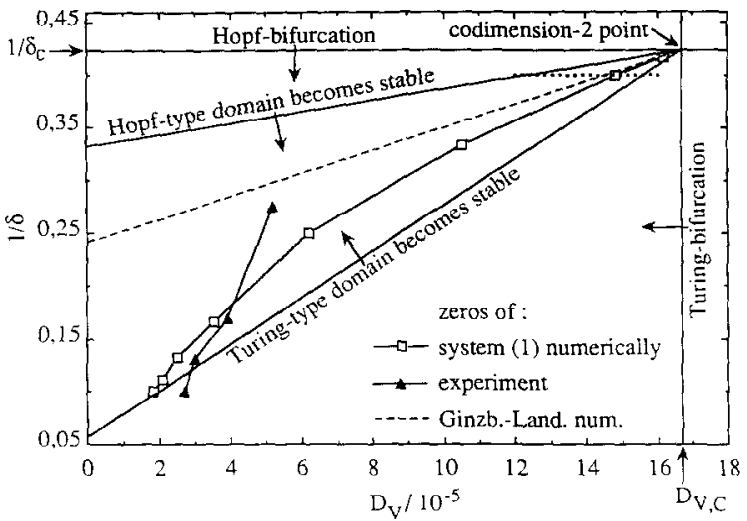

Fig. 8. Zeros of the front velocities in the $D_{v}-1 / \delta$ plane. The asymptotical borders of domain stability can be computed analytically from (3). The dotted line indicates the cross section used for fig. 9.

becomes smaller than $D_{v, \mathrm{c}}$. In the same way the system bifurcates into a homogeneously oscillating state if $D_{v}=D_{v, c}$ and $\delta$ is increased above $\delta_{\mathrm{c}}$. If both these bifurcations occur, the system enters a region of the parameter space where Turing- and Hopf-type domains are possible and transitions between them can be expected. By the method of multiple scales as outlined e.g. in ref. [6] we can derive from system (1) a set of coupled amplitude equations. On the original scales this leads to two coupled Ginzburg-Landau equations,

$$
\begin{aligned}
& \frac{\partial W_{\mathrm{H}}}{\partial t}=m_{\mathrm{H}} W_{\mathrm{H}}+d_{\mathrm{H}} \frac{\partial^{2} W_{\mathrm{H}}}{\partial x^{2}}+g_{\mathrm{H}}\left|W_{\mathrm{H}}\right|^{2} W_{\mathrm{H}} \\
& \quad+K_{\mathrm{H}}\left|W_{\mathrm{T}}\right|^{2} W_{\mathrm{H}}, \\
& \frac{\partial W_{\mathrm{T}}}{\partial t}=m_{\mathrm{T}} W_{\mathrm{T}}+d_{\mathrm{T}} \frac{\partial^{2} W_{\mathrm{T}}}{\partial x^{2}}+g_{\mathrm{T}}\left|W_{\mathrm{T}}\right|^{2} W_{\mathrm{T}} \\
& \quad+K_{\mathrm{T}}\left|W_{\mathrm{H}}\right|^{2} W_{\mathrm{T}} .
\end{aligned}
$$

For the case $\kappa_{\mathrm{j}}=0$ the coefficients are

$$
\begin{aligned}
& m_{\mathrm{H}}=\frac{1}{2}\left(\frac{1}{\delta_{\mathrm{c}}}-\frac{1}{\delta}\right)\left(1-\mathrm{i} \sqrt{\delta_{\mathrm{c}}-1}\right), \\
& m_{\mathrm{T}}=\frac{\left(D_{v, \mathrm{c}}-D_{v}\right) k_{\mathrm{c}}^{2}}{1-\delta_{\mathrm{c}} D_{v, \mathrm{c}} / D_{w}}, \quad k_{\mathrm{c}}^{2}=\frac{1}{\sqrt{D_{v, \mathrm{c}} D_{w}}}-\frac{1}{D_{w}}, \\
& d_{\mathrm{H}}=\frac{1}{2}\left(D_{v, \mathrm{c}}+\frac{D_{w}}{\delta_{\mathrm{c}}}\right)+\frac{\mathrm{i}}{2 \sqrt{\delta_{\mathrm{c}}-1}}\left(-D_{v, \mathrm{c}}+\frac{D_{w}}{\delta_{\mathrm{c}}}\right),
\end{aligned}
$$

$$
\begin{aligned}
& d_{\mathrm{T}}=4 \frac{D_{v, \mathrm{c}}\left(1-\sqrt{D_{v, \mathrm{c}} / D_{w}}\right)}{1-\delta_{\mathrm{c}} D_{v, \mathrm{c}} / D_{w}}, \\
& g_{\mathrm{H}}=-\frac{3}{2}\left(1-\frac{\mathrm{i}}{\sqrt{\delta_{\mathrm{c}}-1}}\right), \quad g_{\mathrm{T}}=-\frac{3}{1-\delta_{\mathrm{c}} D_{v, \mathrm{c}} / D_{w}}, \\
& K_{\mathrm{H}}=2 g_{\mathrm{H}}, \quad K_{\mathrm{T}}=2 g_{\mathrm{T}} .
\end{aligned}
$$

The complex amplitudes $W_{\mathrm{H}}$ and $W_{\mathrm{T}}$ of the domains approximate (1) by

$$
\left(\begin{array}{l}
v \\
w
\end{array}\right) \approx W_{\mathrm{H}} U_{\mathrm{H}} \exp \left(\mathrm{i} \omega_{0} t\right)+W_{\mathrm{T}} U_{\mathrm{T}} \exp \left(\mathrm{i} k_{\mathrm{c}} x\right)+\text { c.c. },
$$

where c.c. denotes the complex conjugate. $U_{\mathrm{H}}$ and $\mathrm{i} \omega_{0}$ are the eigenvector and eigenvalue of the Hopf bifurcation. $U_{\mathrm{T}}$ is the Turing eigenvector and $k_{\mathrm{c}}>0$ the related wavenumber. For the derivation of (3) see refs. $[7,8]$.

In terms of (3) the leading Hopf bifurcation occurs at $\operatorname{Re}\left(m_{\mathrm{H}}\right)=0$, i.e. at $\delta=\delta_{\mathrm{c}}$, whereas the leading Turing bifurcation is located at $m_{\mathrm{T}}=0$, corresponding to $D_{v}=D_{v, \mathrm{c}}$. Both are supercritical and result in stable Hopf respectively Turing states. If $\delta>\delta_{\mathrm{c}}$ and $D_{v}<D_{v, \mathrm{c}}$ (fig. 8), the trivial homogeneous state $\left(W_{\mathbf{H}}\right.$, $\left.W_{\mathrm{T}}\right)=(0,0)$ is unstable due to perturbations both in $W_{\mathrm{H}}$ and in $W_{\mathrm{T}}$. To stabilize a pure Hopf domain ( $\left.W_{\mathrm{H}}=R \mathrm{e}^{\mathrm{i} \omega t}, W_{\mathrm{T}}=0\right)$ in this sector, the growing of $\left|W_{\mathrm{T}}\right|$ has to be suppressed. This is achieved, indeed, by a sufficiently large amplitude $\left|W_{\mathrm{H}}\right|^{2}=-\operatorname{Re}\left(m_{\mathrm{H}}\right) /$ $\operatorname{Re}\left(g_{\mathrm{H}}\right)$, i.e. at a large enough distance from the bifurcation line $\delta=\delta_{\mathrm{c}}$. The condition reads

$m_{\mathrm{T}}+k_{\mathrm{T}}\left|W_{\mathrm{H}}\right|^{2}<0$,

resulting in

$\frac{1}{\delta_{\text {stab,Hopf }}}=\frac{1}{\delta_{\mathrm{c}}}-\frac{1}{2} k_{\mathrm{c}}^{2}\left(D_{v, \mathrm{c}}-D_{v}\right)$.

Considering a pure Turing domain $\left(W_{H}=0\right.$, $W_{\mathrm{T}}=R \mathrm{e}^{\mathrm{i} \Phi}$ ), similarly leads to

$\operatorname{Re}\left(m_{\mathrm{H}}\right)+\operatorname{Re}\left(k_{\mathrm{H}}\right)\left|W_{\mathrm{T}}\right|^{2}<0$,

$\frac{1}{\delta_{\text {stab.Tur }}}=\frac{1}{\delta_{\mathrm{c}}}-2 k_{\mathrm{c}}^{2}\left(D_{v, \mathrm{c}}-D_{v}\right)$.

Hence, Hopf and Turing domains are stable only in part of the sector $D_{\nu}<D_{\nu, c}, \delta>\delta_{\mathrm{c}}$, and we expect stable fronts only within this "bistability" region (fig. 8 ). Deviations from the optimal wavelengths within the domains further reduce the width of this sector 
since they lead to smaller amplitudes. We did not find analytical front-type solutions of (3), so to check the results we compared numerically obtained FFs of (1) and (3) close to $D_{\nu, c}, \delta_{c}$ (fig. 9).

Up to now we have only discussed simple propagation of fronts resulting from certain combinations of Hopf- and Turing-type domains. But one might expect that there are other combinations which do not lead to such a simple result. To induce more complicated behaviour we started with a homogeneous Hopf-type domain in combination with a Turing-type domain which was prepared by a fast FF at small $D_{v}$. If then $D_{v}$ is changed to a value slightly above the estimated zero of the front velocity, first a MF with a small velocity can be observed. But in addition the wavelength within the Turing-type domain slowly increases (fig. 10) and the Hopf-type domain is modulated on a large scale. This finally turns the front back to become a FF. Such a behaviour might be viewed upon as an interaction of two mechanisms:

(a) Propagation of a FF/MF depending on the wavelengths of the domains;

(b) Phase diffusion [6] within the Turing-type domain in the presence of a free boundary tends to increase the wavelength, and the approximately stationary front induces a large-scale modulation of the Hopf-type domain.

So at the bcginning there is a MF bccause the Hopftype domain is "stronger" than the initial Turing-type

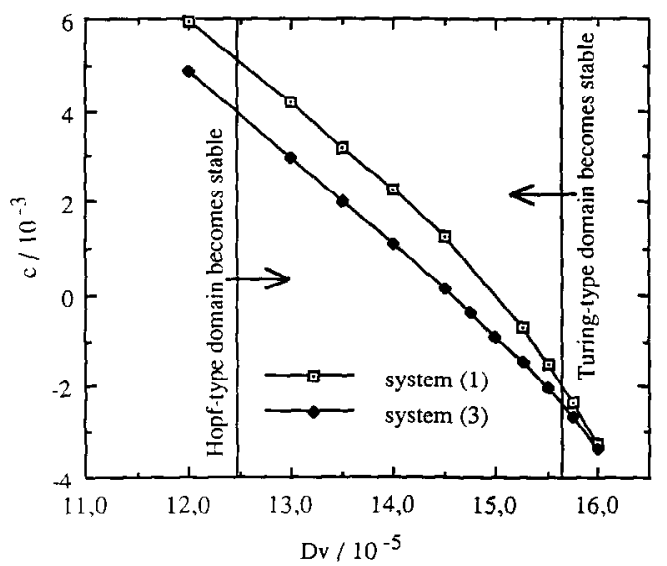

Fig. 9. Comparison of front velocities at $\delta=2.5$. The determination of front velocities can be extended to the ranges of unstable domains in the frame of the Ginzburg-Landau equations, however, for system (1) it was impossible to come to reliable values.

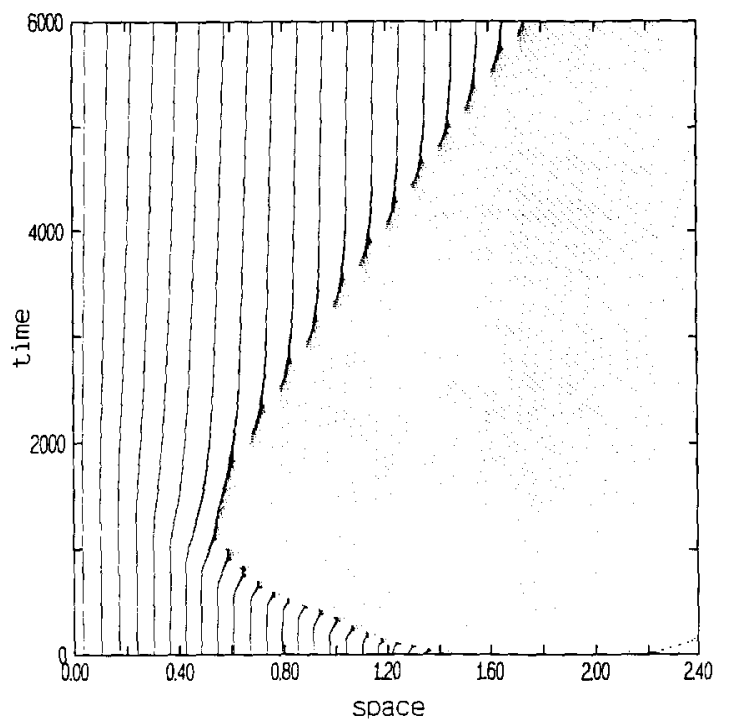

Fig. 10. The same type of diagram as fig. 5. $\delta=9.0$. Here we start the computation with a Turing-typc domain which was left bchind by a FF at $D_{v}=0.5 \times 10^{-5}$, filling approximately half of the system, $A=0.063, A_{\mathrm{T}}=0.070$. At $t=0 D_{v}$ is increased to $1.87 \times 10^{-5}$. After this at first a MF is observed but then phase diffusion turns the front back, generating a new Turing-type domain with $A=0.099, A_{\mathrm{T}}=0.100$ behind the front. The isolated points within the Hopf sector of the plot result from large-scale modulations of the oscillation which can be observed in the case of slowly propagating fronts.

domain, but by changing the wavelengths the Turing-type domain becomes "stronger", so the MF becomes a FF. Moreover, this FF maybe is pushed by the extending Turing-type domain towards the Hopftype domain. Since the short wavelength Turing-type structure is stable, in the case of Neumann boundaries, the wavelength can only be increased in the presence of the front.

Coexistence of different nontrivial patterns has recently been described by Kolodner et al. [9] and by Anderson and Behringer [10]. They observed zones of travelling waves with different wavelengths and phase velocities in a quasi 1-d Rayleigh-Bénard experiment using an ethanol-water mixture. While there is no clear evidence from the work of Anderson and Behringer for fronts between well defined traveling wave domains, Kolodner et al. found such fronts propagating with constant velocity as those described by us.

Similar results have been presented by Willebrand 
et al. [11] in the case of a quasi 1-d (laterally extended) dc-driven gas-discharge system. In addition, these authors found coexisting stationary and traveling patterns separated by a stationary front. Moving Hopf-Turing fronts, however, have so far, to the best of our knowledge, only been described in connection with the oscillator chain discussed in this paper $[1,5]$.

In this context there is extensive theoretical work on patterns governed by a single complex GinzburgLandau equation. In their review of this field, van Saarloos and Hohenberg [12] present several criteria for the existence and stability of fronts and pulses as well as sinks and sources involving different traveling wave states as well as trivial domains. It would be desirable to extend these results to the case discussed in our paper.

In summary we may say that experimental and numerical evidence could be presented for the phenomena of freezing and melting fronts between Turing- and Hopf-type domains in a two-component reaction-diffusion system. Close to the related codimension-two point a pair of coupled GinzburgLandau equations could be derived and was found to be a useful description. We numerically demonstrated the interaction of front propagation and phase diffusion within the domains. It is desirable for the future to study this phenomenon by means of the Ginzburg-Landau equations.
This work has been supported by the Deutsche Forschungsgemeinschaft (DGF).

\section{References}

[1] T. Dirksmeyer, R. Schmeling, J. Berkemeier and H.-G. Purwins, in: NATO ASI series E; Applied sciences, Vol. 183. Patterns, defects, and materials instabilities, eds. D. Walgraef and N.M. Ghonien (Kluwer Academic Publishers, Dordrecht, 1991 ) pp. 91-107.

[2] A.M. Turing, Philos. Trans. R. Soc. 237 (1952) 37.

[3] H. Willebrand, T. Hünteler, F.-J. Niedernostheide, R. Dohmen and H.-G. Purwins, Phys. Rev. A 45 (1992) 10.

[4] F.-J. Niedernostheide, M. Arps, R. Dohmen, H. Willebrand and H.-G. Purwins, Phys. Status Solidi (b) 172 (1992) 249.

[5] J. Berkemeier, T. Dirksmeyer, G. Klempt and H.-G. Purwins, Z. Phys. B 65 (1986).

[6] Y. Kuramoto, Chemical oscillations, waves, and turbulence (Springer, Berlin, 1984).

[7] G. Heidemann, Diplomarbeit, Münster (1992).

[8] M. Bode, Dissertation, Münster (1992).

[9] P. Kolodner, D. Bensimson and C.M. Surko, Phys. Rev. Lett. 60 (1988) 1723 .

[10] K.E. Anderson and R.P. Behringer, Phys. Lett. A 145 (1990) 323.

[11] H. Willebrand, K. Mathiessen, F.-J. Niedernostheide, R. Dohmen and H.-G. Purwins, Contrib. Plasma Phys. 31 (1991) 57.

[12] W. van Saarloos and P.C. Hohenberg, Physica D 56 (1992) 303. 